\title{
Spectroscopic Imaging using Terahertz Time-Domain Signals
}

\author{
Timothy D. Dorney, Richard G. Baraniuk, Daniel M. Mittleman, and Robert D. Nowak \\ Department of Electrical and Computer Engineering, Rice University, Houston, TX 77251-1892 \\ tdorney@ieee.org,richb@rice.edu,daniel@rice.edu,nowak@rice.edu
}

\begin{abstract}
Imaging systems based on terahertz time-domain spectroscopy offer a range of unique modalities due to the broad bandwidth, sub-picosecond duration, and phasesensitive detection of the $\mathrm{THz}$ pulses. Furthermore, an exciting possibility exists to combine spectroscopic characterization and/or identification with imaging because the radiation is broadband in nature. In order to achieve this, novel methods for real-time analysis of $\mathrm{THz}$ waveforms are required. Unfortunately, both the absorption and the phase delay of a transmitted $\mathrm{THz}$ pulse vary exponentially with the sample's thickness. We describe a robust algorithm for extracting both the thickness and the complex index of refraction of an unknown sample. In contrast, most spectroscopic transmission measurements require accurate knowledge of the sample's thickness to determine the optical parameters. We also investigate the limits of our method.
\end{abstract}

\section{Introduction}

The ability to readily explore the electromagnetic spectrum in the far infrared, or terahertz $(\mathrm{THz})$, region of the spectrum has recently been made possible. Novel approaches based on non-linear optics largely overcame the difficulties. One of the most versatile of these recent developments is terahertz time domain spectroscopy (THz-TDS), in which femtosecond optical pulses generate a freely propagating $\mathrm{THz}$ wave via ultrafast gating of a photoconductive switch [1-4]. The resulting electromagnetic pulse is broadband, spanning from below $100 \mathrm{GHz}$ up to several THz. Photoconductive or electrooptic sampling techniques eliminate the need for cumbersome cryogenics for detection of these subpicosecond $\mathrm{THz}$ pulses. Furthermore, these detection schemes are both coherent, in that they provide measurements of the $\mathrm{THz}$ electric field $\mathrm{E}_{\mathrm{THz}}(\mathrm{t})$, rather than the intensity $\left|\mathrm{E}_{\mathrm{THz}}(\mathrm{t})\right|^{2}$. The result is an extremely robust spectrometer with the potential to be both compact and portable.

Adaptations to the THz-TDS system allowed for imaging, using both pixel-by-pixel [4-7] and focal-plane [8-9] methods. These early experiments demonstrated the

We acknowledge support by the Army Research Office, Environmental Protection Agency, and the National Science Foundation. utility of a far infrared imaging system of this sort in applications as diverse as moisture analysis [4-5], package inspection [4-6], biomedical diagnosis [7], and gas sensing [10]. This wide range of applications used a limited scope of signal processing techniques. These most often relied on simple global amplitude or global phase measurements for the encoding of an image. Either the amplitude or the arrival time of the $\mathrm{THz}$ wave transmitted through the sample determined the value of each false-color pixel in the image. Clearly, the utility of the $\mathrm{THz}$ imaging system depends, in many cases, on methods to extract information from the measured waveforms. Consequently, the development of more sophisticated signal processing methods is an important component to enhance and expand the application of the technology.

One of the most interesting possibilities raised by the $\mathrm{THz}$ system is that of spectroscopic analysis combined with pixel-by-pixel imaging. Techniques in other wavelength regimes widely exist [11-13]. Since the $\mathrm{THz}$ radiation generated in the THz-TDS system is broadband, it is possible to implement a spectroscopic imaging system in the far infrared. This is of particular interest because the spectrum of the radiation generated via $\mathrm{THz}-$ TDS spans the region between the high-frequency limit of modern electronic components and the low-frequency limit of most practical lasers and mid-infrared incoherent sources. The implementation of a spectroscopic imaging system based on $\mathrm{THz}-\mathrm{TDS}$, therefore, complements existing multispectral imaging techniques.

Spectroscopic measurements require an analysis method for $\mathrm{THz}$ waveforms. Typically, one measures the transmitted, time-domain waveform both with and without the sample present, and then performs a Fourier deconvolution in order to extract material parameters. The success of this method requires precise knowledge of the thickness of the sample, as both the absorption and the phase delay vary exponentially with sample thickness. Indeed, in many of these measurements, the dominant error in the optical constants arises from uncertainty in the thickness measurement. As a result, the derivation of these constants from the measured electric fields cannot be performed analytically, and numerical methods must be employed [14]. Duvillaret et al. recently described one example of a numerical inversion algorithm for this purpose [15]. As with most spectroscopic methods, this 
algorithm also relies on an accurate knowledge of the sample thickness. Very recently, Duvillaret et al. extended their work to also extract the thickness, but only high index materials were considered [16].

In this work, we propose a new technique to determine simultaneously the thickness and the complex index of refraction of an unknown material. The THz-TDS system provides a time-domain signal of the sample that reveals not only the initial transmission through the material, but also several subsequent internal reflections, given that the material has a high enough index of refraction. Our method is a model-based approach, in which the extraction of the material thickness arises from the analysis of the multiple, internal reflections described by the Fabry-Perot effect. A gradient search minimizes the difference between the model and the measured signals, over a range of thicknesses. At each guessed thickness, we iteratively update the complex index of refraction function to minimize the total error. By introducing a total variation metric to measure the smoothness of the refractive index function at each thickness, we identify the proper thickness and complex index. We investigate both the refractive index limits of our method, and compare the optical parameters of our results to literature data of several different materials.

\section{A model for homogenous, planar materials}

Fourier deconvolution provides a means to determine the frequency response of the sample. To perform this calculation, we require one signal from the system without a sample in situ, and one with a sample. As shown in Fig. 1, the input signal (without sample) is characterized by a single pulse, approximately 1 ps in duration. The waveform with sample shows a similar initial pulse due to the first transmission through the material, but is followed by several smaller pulses due to multiple internal reflections. The output pulse is time shifted, attenuated, and often reshaped compared to the input waveform. These changes form the basis for the information we wish to extract. The Fourier deconvolution is calculated by using the spectrum of the measured signals over the region that has sufficient energy:

$$
H(\omega)=\frac{E_{\text {sample }}(\omega)}{\mathrm{E}_{\text {ref }}(\omega)} .
$$

We generate a model to describe the interaction of the electromagnetic wave with a sample. In Fig. 1, a planar, homogenous material is placed in the pathway. The equations that govern our model are the well known Fresnel equations [17], and are based on a material's complex index of refraction in the frequency domain, $\tilde{n}(\omega)=n(\omega)-j \kappa(\omega)$, where $n(\omega)$ represents the real refractive index, $\kappa(\omega)$ is proportional to the absorption coefficient, and $\omega$ is angular frequency. The function $n(\omega)$ is always greater than 1 , and the function $\kappa(\omega)$ is always nonnegative.

The Fresnel equations at an interface between two layers are:

$$
\begin{aligned}
& t_{a b}(\omega)=\frac{2 \tilde{n}_{a}(\omega)}{\tilde{n}_{a}(\omega)+\tilde{n}_{b}(\omega)} \\
& r_{a b}(\omega)=\frac{\tilde{n}_{b}(\omega)-\tilde{n}_{a}(\omega)}{\tilde{n}_{a}(\omega)+\tilde{n}_{b}(\omega)}
\end{aligned}
$$

where $t_{a b}(\omega)$ is the transmission coefficient of a wave at normal incidence from region $a$ to region $b$, and $r_{a b}(\omega)$ is

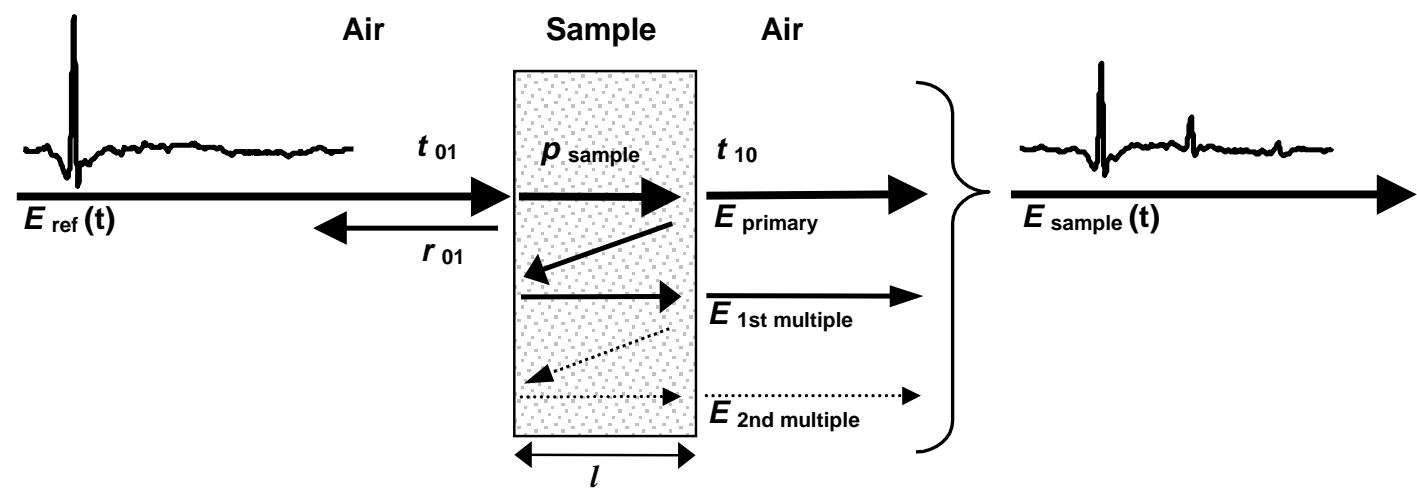

Figure 1. Transmission and reflection pathways for a $\mathrm{THz}$ wave through a planar, homogenous material. We offset the reflection multiples for clarity, and assume all pathways have normal incidence. Our model describes the interactions at each transmission or reflection interface, and the propagation through the material. The input waveform is a single cycle, sub-picosecond pulse. The output signal includes the first transmission through the sample, and two multiples caused by internal reflections. Changes to the input waveform caused by the sample (i.e., time shifts, attenuation, signal reshaping), form the basis for the information used to extract the thickness and optical parameters of the sample. 
the normal reflection in region $a$ at the $a-b$ interface. Conservation of energy constrains $t_{a b}(\omega)+r_{a b}(\omega)=1$. Also, as the electric field moves through a material of thickness $l$, the propagation is governed by:

$$
p_{m}(\omega, l)=\exp \left(\frac{-j \tilde{n}_{m}(\omega) \omega l}{c}\right) \text {. }
$$

The model includes the above propagation equation, but no scattering (e.g., interface roughness).

We consider the first transmission through the material and two multiples caused by internal reflections. All three components are included in the signals recorded by the THz-TDS system. By modeling the THz path both with and without a sample in place, we derive the following frequency domain equation for the model's deconvolution:

$$
\begin{aligned}
\hat{H}(\omega)= & \frac{E_{\text {sample }}(\omega)}{\mathrm{E}_{\text {ref }}(\omega)}=\frac{4 \tilde{n}_{\text {air }}(\omega) \tilde{n}_{\text {sample }}(\omega)}{\left(\tilde{n}_{\text {air }}(\omega)+\tilde{n}_{\text {sample }}(\omega)\right)^{2}} \times \\
& \exp \left(\frac{-j\left(\tilde{n}_{\text {sample }}(\omega)-\tilde{n}_{\text {air }}(\omega)\right) \omega l}{c}\right) \times \\
& \left(1+\sum_{k=1}^{2}\left(r_{10}^{2} p_{\text {sample }}^{2}(\omega, l)\right)^{k}\right)
\end{aligned}
$$

where $l$ is the material thickness, $r_{10}$ is the reflection coefficient, and $p_{\text {sample }}(\omega, l)$ is the propagation coefficient. The complex function $\tilde{n}_{\text {sample }}(\omega)$ and $l$ are the only remaining free variables. Our iterative approach requires that the primary and at least two multiples be present in the measured waveforms, and in the model (i.e., $k=1,2$ ), to solve for the free variables.

\section{Gradient search algorithm}

We define the following error by simply taking the absolute difference between the magnitude and unwrapped phase of the measured data deconvolution versus the model:

$$
\begin{aligned}
& m E R(\omega)=|H(\omega)|-|\hat{H}(\omega)| ; \\
& p E R(\omega)=\angle H(\omega)-\angle \hat{H}(\omega) ; \\
& E R=\sum_{\omega}|m E R(\omega)|+|p E R(\omega)|
\end{aligned}
$$

where $E R$ is the total error.

Our procedure minimizes the total error over a range of reasonable thicknesses by using a gradient descent algorithm on the complex index of refraction functions:

$n(\omega)=n(\omega)+\mu[p E R(\omega)]$

$\kappa(\omega)=\kappa(\omega)+\mu[m E R(\omega)]$

where $\mu=0.01$ provides a good balance between computational overhead and resolution.

After we minimize the total error at a particular thickness, the following equation determines the smoothness, or total variation $(T V)$, of the resulting complex index of refraction [18]:

$T V=\sum D[m]$ where
$D[m]=|n[m-1]-n[m]|+|\kappa[m-1]-\kappa[m]|$.

Total variation provides a good metric since the complex index of refraction is smooth for most solids, but may have an arbitrary shape. This approach fails if sharp spectral features exist (e.g., gases). To speed computation, thickness is scanned first with a coarse stepping distance, and then the scan is refined over a smaller range of thicknesses through two additional passes.

A limited number of trials did not contain a local minimum in the total variation function of the final pass. To counter these poor estimates, we modified the total variation equation to the following:

$T V 2=\sum|D[m]-D[m+1]|$.

The deepest local minimum of the modified total variation, $T V 2$, produced a local minimum for all of our simulation and experimental data.

\section{Results}

We demonstrate the accuracy of our method by estimating the optical parameters for a silicon and lithium niobate (ordinary axis) sample. We also investigate the limits of the THz system.

Figure 2(a) shows the total error plotted over a wide range of thicknesses for a silicon wafer sample. In the next finer scan, Fig. 2(b) shows that the deepest local minimum of the total variation function provides a more accurate estimate of the sample's thickness than the total error deepest local minimum. The deepest local minimum is shallower for the $T V$ metric compared to the total error, but it provides a better estimate of the actual thickness. Total variation also works at low indexes where total error fails.

The wafer's thickness is estimated at $0.54 \mathrm{~mm}$. Measurements made with calipers after the prediction was complete put the expected thickness at approximately $0.51 \pm 0.01 \mathrm{~mm}$. Figure 2(c) contains the real and imaginary index of refraction results at the thickness identified from the TV2 metric. The solid lines indicate the predicted values. The dashed line at 3.418-j0 is the expected real refractive thickness from the literature [19]. The predicted and expected values for the imaginary component are superimposed. The real index is slightly low, since the predicted thickness is slightly high; 


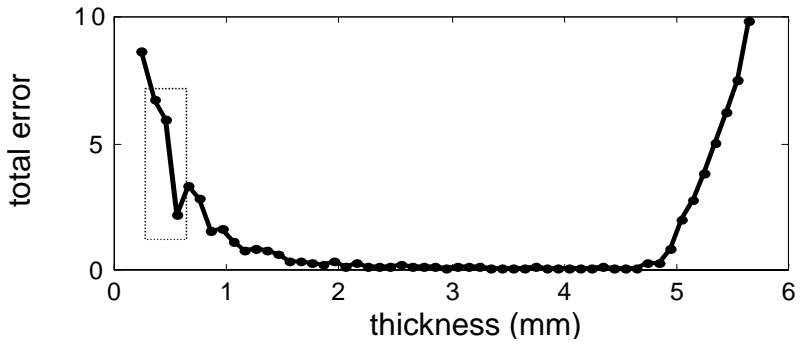

(a)

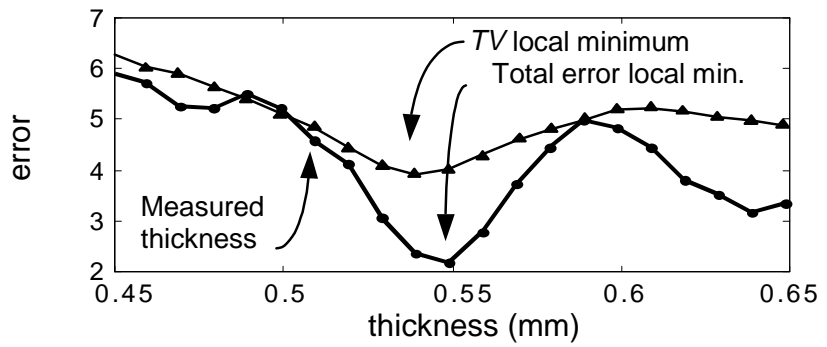

(b)

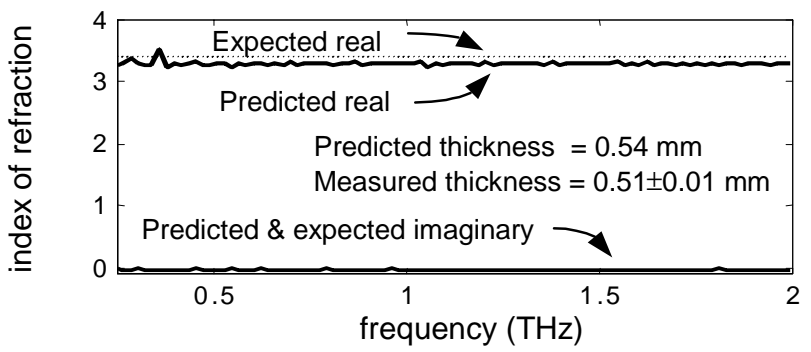

(c)

Figure 2. (a) The total error versus thickness between the modeled and measured signals for a $0.51 \pm 0.01 \mathrm{~mm}$ thick silicon wafer. The box indicates the boundaries for the next graph. (b) Total error versus the total variation metric for an intermediate stepping distance. (c) The corresponding real and imaginary index of refraction for the thickness identified by the modified total variation (TV2) deepest local minimum of the final scan.

however, both the real and imaginary predicted values are independent of frequency as expected. The dominant source of error is sample alignment.

Figure 3(a) shows the total variation metric for a sample of $\mathrm{LiNbO}_{3}$ (ordinary axis). The deepest local minimum occurred at a thickness of $0.44 \mathrm{~mm}$, compared to the measured thickness of $0.50 \pm 0.02 \mathrm{~mm}$. We compare the estimated and literature real index of refraction in Fig. 3(b).

From signal-to-noise measurements of the $\mathrm{THz}$ system, a SNR of 1,500:1 is reasonable. Using simulations and a captured noise signature, we determined the minimum real index that can be resolved by the proposed algorithm. Figure 4 shows the real index for materials versus various thicknesses. The circles indicate test cases that passed using the thicknesses indicated by

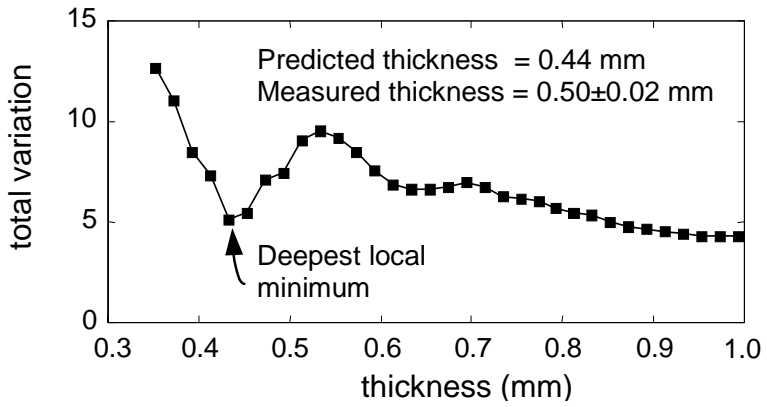

(a)

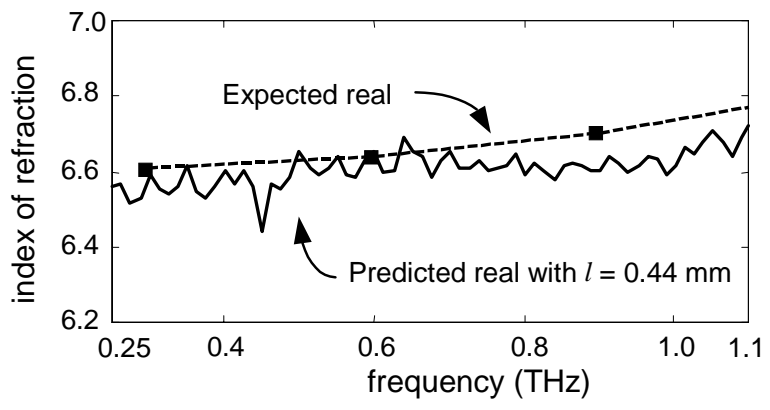

(b)

Figure 3. (a) The total variation measure for $\mathrm{LiNbO}_{3}$ is shown with a $0.02 \mathrm{~mm}$ stepping distance. (b) The corresponding real refractive index for the predicted thickness and the index from the literature are displayed.

the vertical dashed lines and using a non-dispersive $n(\omega)$. The imaginary index was set to zero in these simulations. The shaded area represents the region where the test cases passed. Below this region, the method is limited by signal-to-noise. Above this region, we expected the simulations to pass. The initial step size, however, was too large to produce a local minimum, or the analysis window size $(25 \mathrm{ps})$ limited the number of multiples. Obviously, both the stepping size and analysis window can be changed depending on the material under investigation.

\section{Conclusions}

This paper introduces a method to simultaneously calculate the thickness and complex index of refraction of an unknown homogenous sample using $\mathrm{THz}$ time domain spectroscopy (THz-TDS). The THz-TDS system provides a non-contact, nondestructive approach for investigation. Also, analysis can occur on samples enclosed in standard packaging materials (e.g., cardboard, cellophane, styrofoam) since they are transparent at low $\mathrm{THz}$ frequencies. Our method is limited only by the signal-to-noise ratio of the physical system.

We use a model-based approach, but do not make any direct assumptions about the real or imaginary index 


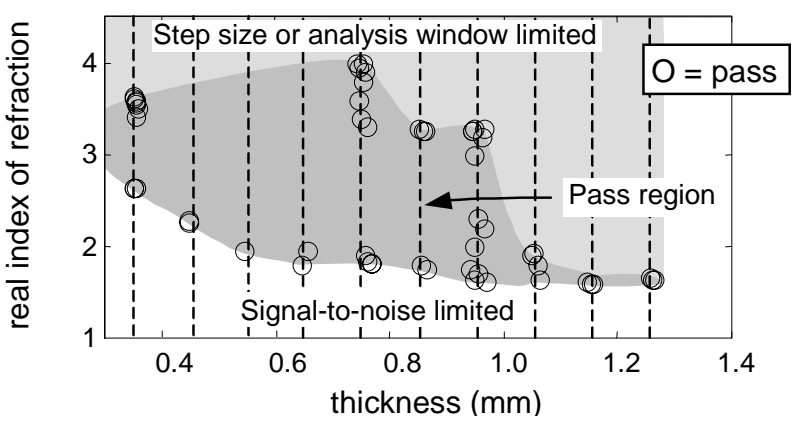

Figure 4. The limits of our method are displayed for a simulated material at a signal-to-noise ratio of 1500:1. The circles indicate the test cases that passed for the thickness indicated by the vertical dashed lines. At each point, the real refractive index was constant across frequency while the imaginary component was zero. The solid areas represent the passing region. The area below the passing region is signal-to-noise limited. The light gray area at the top indicates a region that should pass; however, the initial stepping distance was too large or the analysis window was too small.

of refraction. The algorithm minimizes the absolute difference of the measured waveform's deconvolution and the modeled deconvolution by using a gradient descent algorithm on the complex index of refraction function. The total variation metric provides a measure of the smoothness of the predicted complex index of refraction. By scanning over a range of thicknesses, the deepest local TV2 minimum provides a valid estimate of the material thickness, and consequently, the index of refraction.

Though a single signal pair (reference and signal with sample) is considered in this work, computerized translation of the sample allows for imaging. The addition of simple optics provides a method to achieve a diffraction limited focal spot approximately $250 \mu \mathrm{m}$ in diameter. The THz-TDS system enables nondestructive analysis using a region of the spectrum that is relatively unexplored. This opens a broad new area of investigation. Efforts to determine similar parameters as this paper, but at a lower real index of refraction, are ongoing. The ability to parameterize materials with multiple interfaces or with composite construction is also of interest.

\section{References}

[1] P.R. Smith, D.H. Auston, and M.C. Nuss, "Subpicosecond Photoconducting Dipole Antennas," IEEE J. Quant. Elec., 24, 255-260 (1988).

[2] M. van Exter and D. Grischkowsky, "Characterization of an Optoelectronic Terahertz Beam System," IEEE Trans. Microwave Theory \& Tech., 38, 1684-1691 (1990).
[3] M.C. Nuss and J. Orenstein, "Terahertz Time-Domain Spectroscopy (THz-TDS)," in Millimeter and Submillimeter Wave Spectroscopy of Solids, ed. G. Grüner, (Heidelberg, Germany: Springer-Verlag, 1998), and references therein.

[4] D.M. Mittleman, R.H. Jacobsen, and M.C. Nuss, "T-ray Imaging," IEEE J. Sel. Top. Quant. Elect., 2, 679-692 (1996).

[5] B.B. Hu and M.C. Nuss, "Imaging with terahertz waves," Opt. Lett., 20, 1716-1718 (1995).

[6] D.M. Mittleman, S. Hunsche, L. Boivin, and M.C. Nuss, "T-ray tomography," Opt. Lett., 22, 904-906 (1997).

[7] D.M. Mittleman, M. Gupta, R. Neelamani, R.G. Baraniuk, J.V. Rudd, and M. Koch, "Recent advances in terahertz imaging," Appl. Phys. B, 68, 1085-1094 (1999).

[8] Q. Wu, F.G. Sun, P. Campbell, and X.-C. Zhang, "Dynamic Range of an electro-optic field sensor and its imaging applications," Appl. Phys. Lett., 68, 3324-3326 (1996).

[9] Z.G. Lu, P. Campbell, and X.-C. Zhang, "Free-space electro-optic sampling with a high-repetition-rate regenerative amplified laser," Appl. Phys. Lett., 71, 593-595 (1997).

[10] D.M. Mittleman, R.H. Jacobsen, R. Neelamani, R.G. Baraniuk, and M.C. Nuss, "Gas sensing using terahertz timedomain spectroscopy," Appl. Phys. B, 67, 379-390 (1998).

[11] L. Hui, F. Zhongyu, and Y. Jianqi, "Infrared imaging solar spectrograph at Purple Mountain Observatory," Solar Phys., 185, 69-76 (1999).

[12] K. Sato, T. Sato, H. Sone, and T. Takagi, "Development of a high-speed time-resolved spectroscope and its application to analysis of time-varying optical spectra," IEEE Trans. Inst. Meas., IM-36, 1045-1049 (1987).

[13] R.E. Burge, X.-C. Yuan, J.N. Knauer, M.T. Browne, and P. Charalambous, "Scanning soft X-ray imaging at $10 \mathrm{~nm}$ resolution," Ultramicroscopy, 69, 259-278 (1997).

[14] D. Colton and R. Kress, Inverse Acoustic and Electromagnetic Scattering Theory, $2^{\text {nd }}$ ed. (Heidelberg, Germany: Springer-Verlag, 1998), 2-7.

[15] L. Duvillaret, F. Garet, and J. Coutaz, "A Reliable Method for Extraction of Material Parameters in Terahertz TimeDomain Spectroscopy," IEEE J. Sel. Top. Quant. Elect., 2, 739746 (1996).

[16] L. Duvillaret, F. Garet, and J. Coutaz, "Highly Precise Determination of Both Optical Constants and Sample Thickness in Teraherz Time-Domain Spectroscopy," Appl. Opt., 38, 409415 (1999).

[17] E. Hecht, Optics, $2^{\text {nd }}$ ed. (Reading, Massachusetts. Addison-Wesley, 1987), 92-113.

[18] J.E. Odegard and C.S. Burrus, "Discrete finite variation: a new measure of smoothness for the design of wavelet basis," Proc. of ICASSP, 1467-1470 (1996).

[19] D. Grischkowsky, S. Keiding, M. van Exter, and C. Fattinger, "Far-infrared time-domain spectroscopy with terahertz beams of dielectrics and semiconductors," J. Opt. Soc. Am. B, 7, 2006-2015 (1990). 